\title{
Análisis de la resiliencia y dependencia en deportes de combate y otras modalidades deportivas
}

\author{
Cristina Reche-García ${ }^{a}$, Alejandro Martínez-Rodríguez ${ }^{\text {b* }}$, Magdalena Gómez-Díaz ${ }^{a}$ \\ y Juan José Hernández-Morante ${ }^{a}$
}

${ }^{a}$ Facultad de Enfermería, Universidad Católica San Antonio de Murcia (UCAM), Murcia, España

${ }^{b}$ Facultad de Ciencias, Universidad de Alicante, Alicante, España

Recibido el 1 de octubre de 2019; aceptado el 8 de abril de 2020

\author{
PALABRAS CLAVE \\ Psicología, \\ evaluación, \\ salud, \\ ejercicio
}

\section{KEYWORDS}

Psychology, assessment, health, exercise

\begin{abstract}
Resumen El ejercicio físico (EF) es una práctica sistematizada y orientada a un objetivo (salud, rendimiento, etc.) de actividad física. Dadas las respuestas a nivel fisiológico y cognitivo que genera, este puede influir en diversos factores psicosociales. El objetivo de este estudio es conocer la prevalencia del riesgo de dependencia al EF y de resiliencia por deporte (colectivo, individual o combate) y analizar las diferencias, teniendo en cuenta el sexo, edad, dedicación deportiva y años de experiencia deportiva. Participaron 278 deportistas (194-varones, 84-mujeres). Se administró la Escala de Dependencia del Ejercicio-Revisada y la Escala de Resiliencia. Los resultados mostraron una menor sintomatología de riesgo de dependencia al EF y elevados niveles de resiliencia en deportes de combate frente al resto. En deportes individuales se encuentra una menor resiliencia a medida que van pasando los años de experiencia. En la modalidad colectiva se hallaron diferencias por sexo, mostrando las mujeres mayor resiliencia que los hombres. Los deportes de combate muestran mejores resultados en resiliencia y dependencia al EF.

(c) 2020 Fundación Universitaria Konrad Lorenz. Este es un artículo Open Access bajo la licencia CC BY-NC-ND (http://creativecommons.org/licenses/bync-nd/4.0/).
\end{abstract}

\section{Analysis of resilience and dependence in combat sports and other sports modalities}

Abstract Physical exercise (PE) is a systematized and goal-oriented practice (health, performance, etc.) of physical activity. Given the physiological and cognitive responses that it generates, it can influence various psychosocial factors. The aim of this study is to know risk exercise dependence and resilience prevalence in athletes by sport (collective, individual or combat), and analyze the differences, according to sex, age, sports dedication and years of sports experience. 278 athletes were evaluated (194 men and 84 women) using the Exercise Dependence Scale-Revised and Resilience Scale inventories in the Spanish version. Results showed an important symptomatology of exercise dependence risk and higher levels of resilience in combat sports in comparison with team sports or individual sports. There was a

\footnotetext{
* Autor para correspondencia.

Correo electrónico: amartinezrodriguez@ua.es
}

https://doi.org/10.14349/sumapsi.2020.v27.n2.1

ISSN 0121-4381, ISSN-E 2145-9797/@ 2020 Fundación Universitaria Konrad Lorenz. Este es un artículo Open Access bajo la licencia CC BY-NCND (http://creativecommons.org/licenses/by-nc-nd/4.0/). 
significant negative correlation between resilience and years of expertise in individual sports athletes. Results from team sports athletes presented significant differences between men and women in resilience, increased in women. Sport combat practice showed better results in resilience and exercise dependence.

(c) 2020 Fundación Universitaria Konrad Lorenz. This is an open access article under the CC BYNC-ND license (http://creativecommons.org/licenses/bync-nd/4.0/).

La naturaleza de la resiliencia se relaciona con perfiles de personalidad equilibrados y saludables psicológicamente (Friborg, Barlaug, Martinussen, Rosenvinge \& Hjemdal, 2005; Manzano-García, Ayala-Calvo, 2013); y con personas capaces de cambiar de forma flexible sus respuestas afectivas y fisiológicas, para adecuarlas a las demandas del ambiente (Menezes-de Lucena Carvalho, FernándezCalvo, Hernández-Martín, Ramos-Campos \& Contador-Castillo, 2006; Waugh, Thompson \& Gotlib, 2011). El desarrollo de la resiliencia puede mejorar el rendimiento deportivo y viceversa (Chacón-Cuberos, Castro-Sánchez, Espejo-Garcés \& Zurita-Ortega, 2016). La resiliencia es un concepto que ha mostrado una importancia creciente en los últimos años dentro del ámbito de los deportes (Zurita-Ortega, ChacónCuberos, Cofre-Bolados, Knox \& Muros, 2018). En la bibliografía más reciente se han encontrado prevalencias de hasta un $24.8 \%$ de resiliencia elevada en deportistas de distintas modalidades (Reche, Martínez-Rodríguez \& Ortín, 2019), un 17\% en esgrimistas (Reche, Gómez-Díaz, Martínez-Rodríguez \& Tutte, 2018) y un $8.5 \%$ en hockey (Tutte \& Reche, 2016). En el caso del fútbol, balonmano y esquí, un estudio refiere que los esquiadores poseen puntuaciones de resiliencia ligeramente más elevadas, siendo los futbolistas los que presentan menor puntuación (Chacón-Cuberos et al., 2016).

Otro factor que aparece en el deporte, pero en forma de trastorno, es la dependencia del ejercicio, identificada con aproximaciones a los componentes establecidos en el DSM- 5 para las adicciones conductuales y con las consecuencias colaterales derivadas (Márquez \& De la Vega, 2015); es decir, con un malestar clínicamente significativo en diversas áreas de la vida del sujeto, con riesgo de manifestar alteraciones psicológicas o psicopatológicas, físicas y sociales; con consecuencias adversas y recurrentes relacionadas con el ejercicio continuado y su retirada, y que afectan la calidad de vida, bienestar y el rendimiento deportivo del individuo (Sicilia, Alías-García, Ferriz \& Moreno-Murcia, 2013). La evidencia científica más reciente ha encontrado prevalencias de hasta un $8.3 \%$ de riesgo por dependencia al ejercicio físico en deportistas de equipo e individuales (Reche, et al., 2019), y con el paso de los años se han observado diferencias entre distintos deportes. Ya en 1993 revelaron que las bailarinas tenían más dependencia que las corredoras y jugadoras de hockey sobre hierba (Pierce, Daleng \& McGowan, 1993); en 1998, encontraron en levantadores de pesas de competición mayores niveles de dependencia que los informados anteriormente por otros deportistas de resistencia (Pierce \& Morris, 1998); en 2002 aparecen prevalencias del $30.4 \%$ en grupos de triatletas (Blaydon $\&$ Lindner, 2002), cifras elevadas en comparación con las prevalencias generales; y más recientemente, se observa cómo los triatletas presentan mayor puntuación que ciclistas, nadadores y corredores, con diferencias significativas en dependencia entre triatletas y corredores y ciclistas y corredores (Latorre, Jiménez, Párraga \& García-Pinillos, 2016). Así que recientemente se ha recomendado realizar análisis de dependencia al ejercicio físico teniendo en cuenta diferentes tipos de deportes de los ya habitual- mente estudiados a lo largo de la historia de esta adicción conductual (Ortiz \& Arbinaga, 2017).

Tanto la resiliencia como la dependencia al ejercicio físico han sido constructos estudiados en función de distintas modalidades deportivas, individual y equipos (ChacónCuberos et al., 2016; Reche, Martínez-Rodríguez \& Ortín, 2015), sin encontrar diferencias (Modolo, Mello, Giménez, Tufik \& Antunes, 2009; Reche et al., 2015; Reche, De Francisco, Martínez-Rodríguez \& Ros-Martínez, 2018), pero en ningún caso ha sido atendida la peculiaridad de los deportes de combate. Sin embargo, es más probable que la participación en ciertas actividades dé como resultado una mayor dependencia del ejercicio que en otras.

Los deportes de combate no se encuentran tan masificados como los deportes colectivos, pero sí presentan una notable representación dentro de los deportes de práctica individual, pudiendo configurarse como deportes con características específicas no solo técnicas, tácticas o de condición física, sino también psicológicas (Ruiz-Barquín, 2012).

Los deportes de combate representan el $12 \%$ de las medallas españolas de los Juegos Olímpicos. Se trata de disciplinas deportivas en las que dos oponentes se enfrentan con el objetivo de derribar o vencer al rival en un enfrentamiento de múltiples asaltos de corto espacio con intensidad máxima (Mata-Ordoñez, Sánchez-Oliver \& Domínguez, 2018).

Con frecuencia los deportistas de combate indican que su práctica produce efectos psicosociales diferentes de los producidos por otros deportes (y mayores que estos), promoviendo bienestar psicológico, pero con procesos y efectos distintos (González-Hernández, 2011).

Siguiendo a Iglesias, Gasset, González y Anguera (2010), los deportes de combate se caracterizan por la interacción permanente en las situaciones de juego. No existe una red que divida los terrenos ni una posesión de balón que determine la acción ofensiva. Ambos rivales interactúan solapando sus amenazas ofensivas a sus opciones defensivas, y estas conductas se modifican en función de los factores ambientales específicos. Hernández-García y Torres-Luque (2011) además los caracterizan señalando que son de habilidad abierta, con un nivel de incertidumbre muy elevado, por tener al oponente cuerpo a cuerpo. Presentan una gran intensidad del esfuerzo, con enormes exigencias físicas, fisiológicas, técnico-tácticas y psicológicas ante el enfrentamiento; y muestran dificultad de prescripción-control del entrenamiento y la competición, y mucho más aún de alto nivel.

Sin embargo, no se ha estudiado cómo la práctica de deportes de combate se relaciona con elevados niveles de resiliencia o con sintomatología de riesgo de dependencia. Con la finalidad de observar qué deporte podría contribuir a una mejor salud psicológica en el deportista por presencia o ausencia de los constructos referidos, se propone conocer la prevalencia del riesgo de dependencia al EF y de resiliencia por deporte (colectivo, individual o combate), y analizar las diferencias, teniendo en cuenta sexo, edad, dedicación deportiva y años de experiencia deportiva. El conocimiento de la existencia diferencial de sintomatología conductual según el tipo de deporte practicado permite 
discriminar a los deportistas más vulnerables a padecer de una dependencia al ejercicio físico y a los más fortalecidos y resilientes, identificando deportes que pudieran ser más saludables sin riesgos.

\section{Método}

\section{Participantes}

El estudio se llevó a cabo con 278 deportistas de distintas disciplinas; 194 varones y 84 mujeres (edad: 18-60 años, $M=23, D T=7$ ) en periodo de entrenamiento. Para constituir la muestra, se han seguido datos del Anuario de Estadísticas Deportivas del Instituto Nacional de Estadística (2019), que muestran la representatividad en España de hábitos deportivos por modalidades. De los participantes en el estudio, un $50.7 \%$ son junior (18-20 años de edad) y un $49.3 \%$ senior ( $\geq 20$ años de edad). Los atletas presentan distintos niveles deportivos, siendo un $64.4 \%$ aficionados y un $35.6 \%$ élite.

El $30.2 \%$ de la muestra de estudio eran participantes de deportes individuales como atletismo. El 59.7\% de deportistas practicaban deportes colectivos como fútbol o baloncesto. El $10.1 \%$ de deportistas practicaban deportes de combate como esgrima, karate o taekwondo, entre otros. El $20.5 \%$ de los deportistas habían practicado su deporte por menos de cuatro años y el 79.5\%, durante cuatro o más años. Los entrenamientos semanales abarcaban el rango entre $1-16$ sesiones $(M=4, D T=2)$. El $20.5 \%$ practicaba ejercicio físico por lo menos una hora/semana y hasta cuatro, y el $79.5 \%$ restante más de cuatro horas y hasta un máximo de 56 . El $4 \%$ practicaban deporte menos de un año, entre uno y dos el $5 \%$, de tres a cinco el $12 \%$, de seis a 10 el $22 \%$ y más de 10 el $58 \%$.

\section{Instrumentos}

Se aplicaron cuestionarios de recopilación de datos sociodemográficos y de actividad deportiva, sesiones de entrenamiento y años de experiencia.

Se administró la Escala de Resiliencia (Ruiz-Barquín, De la Vega-Marcos, Poveda, Rosado \& Serpa, 2012; adaptación de Wagnild \& Young, 1993) adaptada al español en fútbol, y confiable en una muestra de esgrimistas españoles y de judokas uruguayos (Reche \& Ortín, 2013; Reche, Tutte \& Ortín, 2014), con un $\alpha$ de Cronbach de .89 en ambos casos. En nuestro estudio la escala presenta una consistencia interna ( $\alpha$ de Cronbach) de .82. Se trata de una escala formada por 25 ítems escritos de forma positiva, y con un formato de respuesta tipo Likert de siete puntos. Se evalúa el grado de resiliencia individual y la perspectiva de resiliencia como una característica de personalidad positiva que favorece la adaptación. La escala permite obtener una puntuación global de resiliencia (a mayor puntuación, más elevada se considera) y una puntuación para cada uno de los factores: Competencia personal, que indica autoconfianza-independencia-decisión-invencibilidad-poderío-ingenio-perseverancia; y Aceptación de uno mismo y de la vida, representando adaptabilidad-balance-flexibilidad-perspectiva de vida estable. Estos dos factores consideran las características de resiliencia (Salgado, 2005): Ecuanimidad-Perseverancia-Confianza en sí mismo-Satisfacción personal-Sentirse bien solo. En nuestro estudio la dimensión Competencia personal alcanza un $\alpha$ de Cronbach de .85, mientras que la Aceptación de uno mismo y de la vida, .44.

En cuanto a las puntuaciones, pueden oscilar en un rango comprendido entre 25-175, estableciéndose diferentes rangos: puntuaciones $\geq 147$ indican un nivel elevado de resiliencia; de 121-147 indican niveles moderados, y $<121$ indican poca capacidad de resiliencia (Reche et al., 2014).

Para evaluar la dependencia al ejercicio físico se utilizó la Escala Revisada de Dependencia del Ejercicio (EDS-R; Sicilia \& González, 2011), la cual consta de 21 ítems y hace referencia a creencias y comportamientos que han ocurrido en los últimos tres meses. Los sujetos responden en una escala tipo Likert de 1 a $6(0=$ nunca; $6=$ siempre $)$. Esta escala conceptualiza la dependencia al ejercicio físico apoyándose en los criterios de dependencia a sustancias del DSM-IV (American Psychiatric Association, 1994). Las subescalas que la conforman son: Tolerancia, Abstinencia, Efectos deseados, Falta de control, Reducción de otras actividades, Tiempo y Continuación. Mediante el análisis de fiabilidad del instrumento se obtuvieron coeficientes de consistencia ( $\alpha$ de Cronbach) superiores a .70, salvo para la subescala Reducción de otras actividades. Un valor $\alpha$ de .73 para la Tolerancia, .85 para la Abstinencia, .83 para Efectos deseados, .78 para la Falta de control, .84 para el Tiempo empleado, .68 para la Reducción de otras actividades y .81 para la Continuación (Sicilia \& González-Cutre, 2011). El valor $\alpha$ para la consistencia global de la escala fue de .92 (Hausenblas \& Downs, 2002), presentando aquellos sujetos con una mayor puntuación EDS-R más síntomas de dependencia al ejercicio (Downs, Hausenblas \& Nigg, 2004). En este estudio la escala presenta una consistencia interna ( $\alpha$ de Cronbach) de .89. En Abstinencia .79, en Continuación .80, en Tolerancia .87, en Falta de control .78, en Reducción de otras actividades .66, en Tiempo .74 y en Efectos deseados.80.

El EDS-R permite clasificar a los individuos en: riesgo de dependencia; sintomáticos, pero no dependientes; y asintomáticos no dependientes. El grupo con riesgo de dependencia está formado por individuos con puntuaciones > 5 en tres criterios. El grupo de sintomáticos no dependientes está formado por aquellos con puntuaciones de 3-4 en tres o más criterios o que obtienen puntuaciones de 5-6 combinadas con puntuaciones de 3-4 en tres criterios, sin llegar a los requisitos para ser incluidos en el grupo de riesgo por dependencia. El grupo asintomático no dependientes está formado por individuos con una puntuación mínima de 1-2 en al menos tres criterios, sin alcanzar los requisitos de incorporación en el grupo sintomáticos no dependientes.

\section{Procedimiento}

Para la recogida de datos y aplicación de los cuestionarios se solicitaron los permisos pertinentes a diferentes federaciones y clubes de la Comunidad Valenciana y de la Región de Murcia. El cuestionario fue entregado y aplicado por psicólogos del deporte durante los entrenamientos de los deportistas, de forma individual y voluntaria. Se solicitó mediante un consentimiento informado la autorización y deseo de colaboración con la investigación. Los criterios de exclusión fueron: ser menores de 18 años y presentar pruebas incompletas (falta de datos esenciales y/o ítems necesarios). El procedimiento fue aprobado por el Comité de Ética de la Universidad Católica San Antonio de Murcia.

\section{Análisis estadístico}

Se trata de un estudio descriptivo con carácter transversal. Se realizaron análisis descriptivos (frecuencias y porcentajes para determinar la prevalencia de los constructos evaluados y sus dimensiones, en deportes de combate $\mathrm{u}$ otros, distinguiendo por edad/sexo/sesiones entreno/ años experiencia); se utilizaron tablas de contingencia y la prueba de Chi-cuadrado. Los distintos deportes evaluados 
(colectivos, individuales y deportes de combate) fueron analizados mediante varianzas, con un test de Bonferroni para realizar los análisis post hoc, por el tamaño muestral, habiendo comprobado la normalidad de la distribución de la muestra con la prueba de Kolmogorov-Smirnov.

El análisis se realizó utilizando el software SPSS v.24, y el nivel de significación se estableció $p<.05$. Los tamaños de los efectos se calcularon utilizando el estadístico eta-cuadrado parcial $\left(\mathrm{np}^{2}\right)$ para establecer la significación sustantiva de las diferencias encontradas. Se establecieron como indicadores: pequeño (.20), mediano (.50) y grande (.80) (Richardson, 2011).

\section{Resultados}

Los análisis descriptivos determinaron una prevalencia del $6.5 \%$ de riesgo de dependencia al ejercicio físico y un
$27 \%$ de elevada resiliencia en la totalidad de los deportistas. Más de la mitad de los participantes del estudio presentaron moderada resiliencia (54\%) y sintomatología, pero no dependencia al ejercicio (59\%).

En la tabla 1 se muestra la comparación por modalidades deportivas (colectivo/individual/combate). El análisis de varianza (ANOVA) mostró diferencias en las puntuaciones totales del EDS-R con una menor sintomatología de dependencia al ejercicio físico en deportes de combate frente a deportes de equipo e individuales (figura 1), con un tamaño del efecto grande $\left(n p^{2}=.93\right)$. Se encontraron diferencias significativas en las subescalas del EDS-R; Continuación $(p=.00)$ entre colectivo e individual; Tolerancia $(p=.04)$ en colectivo e individual; Falta de control $(p=.00)$ en colectivo e individual; Reducción $(p=.01)$ entre colectivo y combate, y entre individual y combate; Tiempo $(p=.03)$

Tabla 1 ANOVA-Comparación entre grupos por modalidad deportiva.

\begin{tabular}{|c|c|c|c|c|c|c|c|}
\hline & & Media & DS & $F$ & Sig. & IC (95\%) & $n p^{2}$ \\
\hline \multirow[t]{3}{*}{ EDS-R Total } & Colectivo $(n=166)$ & 71.09 & 15.63 & 6.06 & .00 & 68.7-73.47 & .93 \\
\hline & Individual $(n=84)$ & 64.28 & 16.68 & & & $60.71-67.85$ & \\
\hline & Combate $(n=28)$ & 63.57 & 20.00 & & & $55.81-71.33$ & \\
\hline \multirow[t]{3}{*}{ Abstinencia } & Colectivo $(n=166)$ & 12.38 & 3.29 & 1.31 & .26 & $11.88-12.88$ & .75 \\
\hline & Individual $(n=84)$ & 11.70 & 3.27 & & & $11.00-12.40$ & \\
\hline & Combate $(n=28)$ & 11.82 & 3.48 & & & $10.47-13.17$ & \\
\hline \multirow[t]{3}{*}{ Continuación } & Colectivo $(n=166)$ & 10.04 & 3.63 & 5.51 & .00 & $9.49-10,59$ & .89 \\
\hline & Individual $(n=84)$ & 8.36 & 3.91 & & & $7.52-9.20$ & \\
\hline & Combate $(n=28)$ & 9.82 & 4.36 & & & $8.13-11.51$ & \\
\hline \multirow[t]{3}{*}{ Tolerancia } & Colectivo $(n=166)$ & 9.95 & 3.71 & 3.22 & .04 & $9.39-10.51$ & .28 \\
\hline & Individual $(n=84)$ & 8.55 & 4.58 & & & $7.57-9.53$ & \\
\hline & Combate $(n=28)$ & 9.78 & 5.03 & & & $7.83-11.73$ & \\
\hline \multirow[t]{3}{*}{ Falta de control } & Colectivo $(n=166)$ & 10.19 & 3.46 & 6.37 & .00 & $9.66-10.72$ & .88 \\
\hline & Individual $(n=84)$ & 8.57 & 3.45 & & & $7.83-9.31$ & \\
\hline & Combate $(n=28)$ & 9.10 & 3.71 & & & $7.66-10.54$ & \\
\hline \multirow[t]{3}{*}{ Reducción } & Colectivo $(n=166)$ & 8.16 & 3.02 & 4.63 & .01 & $7.70-8.62$ & .58 \\
\hline & Individual $(n=84)$ & 7.28 & 3.14 & & & $6.61-7.95$ & \\
\hline & Combate $(n=28)$ & 6.57 & 2.68 & & & $5.53-7.61$ & \\
\hline \multirow[t]{3}{*}{ Tiempo } & Colectivo $(n=166)$ & 11.50 & 3.24 & 3.31 & .03 & $11.01-11.99$ & .87 \\
\hline & Individual $(n=84)$ & 11.53 & 3.73 & & & $10.73-12.33$ & \\
\hline & Combate $(n=28)$ & 9.75 & 3.64 & & & $8.34-11.16$ & \\
\hline \multirow[t]{3}{*}{ Efectos deseados } & Colectivo $(n=166)$ & 8.84 & 3.40 & 5.21 & .00 & $8.32-9.36$ & .84 \\
\hline & Individual $(n=84)$ & 8.26 & 3.23 & & & 7.57-8.95 & \\
\hline & Combate $(n=28)$ & 6.71 & 2.86 & & & $5.60-7.82$ & \\
\hline \multirow[t]{3}{*}{ Resiliencia total } & Colectivo $(n=166)$ & 133.34 & 17.29 & 5.52 & .00 & $130.61-135.87$ & .85 \\
\hline & Individual $(n=84)$ & 135.16 & 18.35 & & & $131.24-139.08$ & \\
\hline & Combate $(n=28)$ & 145.39 & 18.45 & & & $138.24-152.54$ & \\
\hline \multirow[t]{3}{*}{ Competencia personal } & Colectivo $(n=166)$ & 92.75 & 12.33 & 4.33 & .01 & $90.87-94.63$ & .74 \\
\hline & Individual $(n=84)$ & 94.82 & 13.26 & & & $91.98-97.66$ & \\
\hline & Combate $(n=28)$ & 100.10 & 10.91 & & & $95.87-104.33$ & \\
\hline \multirow[t]{3}{*}{ Aceptación de uno mismo } & Colectivo $(n=166)$ & 31.12 & 4.74 & .86 & .42 & $30.40-31.84$ & .19 \\
\hline & Individual $(n=84)$ & 30.45 & 5.11 & & & $29.36-31.54$ & \\
\hline & Combate $(n=28)$ & 31.67 & 4.65 & & & 29.87-33.47 & \\
\hline
\end{tabular}


entre colectivo y combate, e individual y combate; y Efectos deseados $(p=.00)$ entre colectivo y combate, e individual y combate.

Así mismo, en la escala de Resiliencia, las diferencias significativas se presentaron en su totalidad $(p=.00)$ con un tamaño del efecto grande $\left(n \mathrm{p}^{2}=.85\right)$ apuntando que los deportes de combate presentan mayor resiliencia frente a los individuales y de equipo (tabla 1 y figura 2). En la dimensión Competencia personal se encontraron diferencias $(p=.01)$. En este caso los deportes de combate muestran valores significativamente superiores frente a los deportes colectivos.

En primer lugar, respecto de los practicantes de deportes de combate, los datos indicaron que el $57 \%$ de las mujeres presentaron niveles elevados de resiliencia frente a un $47.6 \%$ de los hombres, diferencias que no fueron estadísticamente significativas. La categoría de edad a la que pertenecen los deportistas tampoco parece influir en los niveles de resiliencia, ya que los junior y los senior tenían valores similares. Igual sucede con las sesiones de entrenamiento semanales y los años de práctica deportiva (tabla 2). En el caso de la dependencia al ejercicio físico en los deportistas de combate, no se encuentran diferencias significativas por sexo, ni categoría de edad, ni por número de sesiones, ni por años de experiencia. Aparecen mayores puntuaciones de sintomatología en las mujeres, en los senior, en los que realizaban mayores sesiones de entrenamiento y practicaban su deporte durante menos años.

Ahora bien, al estudiar los niveles de resiliencia en deportistas individuales, sin contar con los de combate, tampoco se encontraron diferencias significativas en función

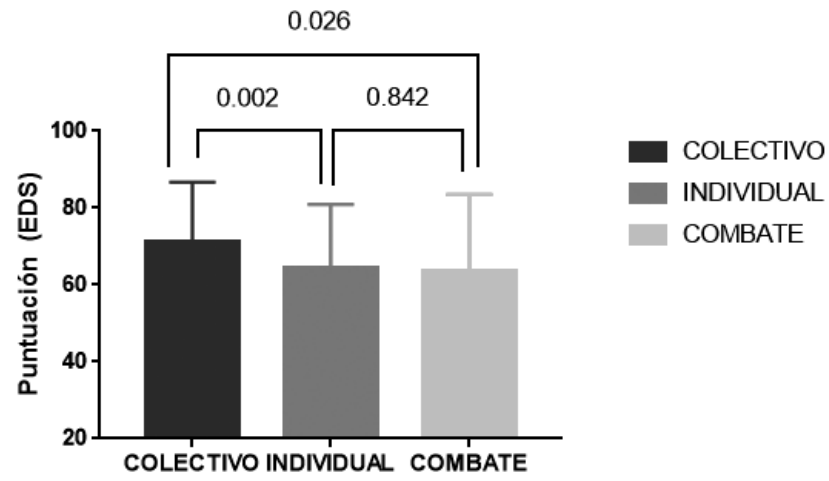

Figura 1. Diferencias en las puntuaciones del EDS en función de los distintos deportes.

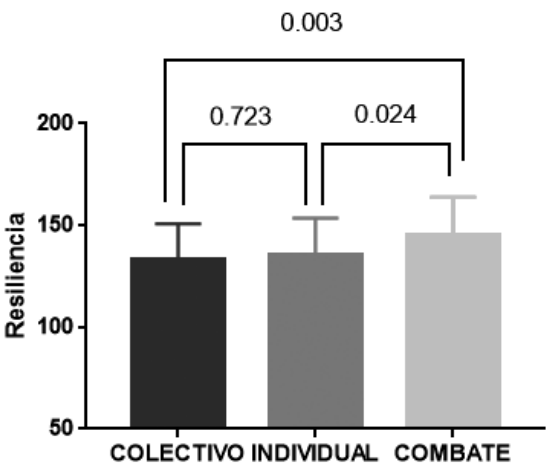

COLECTIVO INDIVIDUAL COMBATE

Figura 2. Diferencias en las puntuaciones de la Escala de Resiliencia en función de los distintos deportes. del sexo, categoría de edad, sesiones de entrenamiento y número de sesiones semanales; pero sí en función de los años de experiencia deportiva $(p=.02)$. El $26.8 \%$ de los deportistas con una experiencia deportiva menor de cuatro años presenta mayores niveles de resiliencia que los deportistas con cuatro años o más, con el 17.9\%. En el caso del riesgo de dependencia al ejercicio físico, se presentó una sintomatología similar en función del sexo, categoría de edad, sesiones de entrenamiento y años de práctica, siendo los hombres, los junior y los que practican más sesiones semanales de entrenamiento, y durante más años, los que mayor sintomatología presentan (tabla 3).

Finalmente, en deportes colectivos los resultados reportaron mayores niveles de resiliencia en las mujeres que en los hombres $(p=.03)$, un $35.8 \%$ frente al $19.5 \%$. En cuanto a la categoría de edad, sesiones de entrenamiento y años de práctica, no aparecen diferencias significativas. En el caso del riesgo de dependencia al ejercicio físico no se encuentran diferencias significativas en ninguna de las variables estudiadas, siendo las puntuaciones mayores en mujeres, junior, los que practican más sesiones a la semana y los que han practicado su deporte por menos años (tabla 4).

\section{Discusión}

Este estudio tuvo como objetivo conocer la prevalencia del riesgo de dependencia al EF y de resiliencia por deporte (colectivo, individual o combate), y analizar las diferencias, teniendo en cuenta el sexo, edad, dedicación deportiva y años de experiencia deportiva. Los resultados mostraron una destacable menor sintomatología de riesgo de dependencia al ejercicio físico en deportes de combate frente a deportes colectivos e individuales; en todas sus dimensiones, a excepción de la que se refiere a la manifestación del síndrome de abstinencia. Además, deportistas más resilientes (caracterizados por confianza en sí mismos, independencia, decisión, invencibilidad, poderío, ingenio y perseverancia) entre los practicantes de deportes de combate, frente a deportistas de equipo e individuales.

Los niveles elevados de resiliencia aparecieron en un $27 \%$ de los deportistas. Siguiendo la evidencia científica no aparecen diferencias por sexo en resiliencia en distintos deportes colectivos-individuales-combate (Reche et al., 2019), tampoco específicamente en nadadores (Campos, ValdiviaMoral, Zagalaz, Ortega \& Romero, 2017). Sin embargo, cuando se separó la muestra por deportistas de equipo, individuales y de combate, se obtuvo que en la modalidad de equipo las deportistas presentaron significativamente puntuaciones más elevadas que los hombres. Quizá el trabajo en equipo, dadas sus exigencias y características propias, favorezca el cambio flexible de respuestas para la adecuación a las demandas en la participación en su deporte, utilizando recursos individuales combinados para adaptarse de forma positiva a la adversidad (Morgan, Fletcher \& Sarkar, 2013). Siendo las mujeres más sensibles a estas características. Siguiendo la recomendación de Moreno-Murcia, Belando, Huéscar y Torres (2017) se podría intervenir para maximizar los beneficios, a nivel de salud y de satisfacción con la vida, que tienen la práctica física y deportiva en la mujer. 
Tabla 2 Contingencia de resiliencia y dependencia en deportes de combate, y sexo/edad/sesiones de entreno/experiencia deportiva $(\%, \mathrm{~N})$.

\begin{tabular}{|c|c|c|c|c|c|c|}
\hline & \multicolumn{3}{|c|}{ Resiliencia deportes de combate } & \multicolumn{3}{|c|}{ EDS-R Dependencia al ejercicio } \\
\hline & $<$ 121. Poca & 121-147. Moderada & > 147. Elevada & $\begin{array}{c}\text { Asintomático } \\
\text { no dependiente }\end{array}$ & $\begin{array}{c}\text { Sintomático } \\
\text { no dependiente }\end{array}$ & $\begin{array}{c}\text { Riesgo de } \\
\text { dependencia }\end{array}$ \\
\hline Hombres $(n=21)$ & $\begin{array}{c}9.5 \% \\
2\end{array}$ & $\begin{array}{c}42.9 \% \\
9\end{array}$ & $\begin{array}{c}47.6 \% \\
10\end{array}$ & $\begin{array}{c}52.4 \% \\
11\end{array}$ & $\begin{array}{c}42.9 \% \\
9\end{array}$ & $\begin{array}{c}4.8 \% \\
1\end{array}$ \\
\hline Mujeres $(n=7)$ & $\begin{array}{c}0 \% \\
0\end{array}$ & $\begin{array}{c}42.9 \% \\
3\end{array}$ & $\begin{array}{c}57.1 \% \\
4\end{array}$ & $\begin{array}{c}28.6 \% \\
2\end{array}$ & $\begin{array}{c}57.1 \% \\
4\end{array}$ & $\begin{array}{c}14.3 \% \\
1\end{array}$ \\
\hline Junior $(n=11)$ & $\begin{array}{c}0 \% \\
0\end{array}$ & $\begin{array}{l}45.5 \% \\
5\end{array}$ & $\begin{array}{c}54.5 \% \\
6\end{array}$ & $\begin{array}{c}45.5 \% \\
5\end{array}$ & $\begin{array}{c}54.5 \% \\
6\end{array}$ & $\begin{array}{c}0 \% \\
0\end{array}$ \\
\hline Senior $(n=17)$ & $\begin{array}{c}11.8 \% \\
2\end{array}$ & $\begin{array}{l}41.2 \% \\
7\end{array}$ & $\begin{array}{c}47.1 \% \\
8\end{array}$ & $\begin{array}{c}47.1 \% \\
8\end{array}$ & $\begin{array}{c}41.2 \% \\
7\end{array}$ & $\begin{array}{c}11.8 \% \\
2\end{array}$ \\
\hline $\begin{array}{l}\text { Hasta cuatro sesiones } \\
(n=19)\end{array}$ & $\begin{array}{c}10.5 \% \\
2\end{array}$ & $\begin{array}{c}47.4 \% \\
9\end{array}$ & $\begin{array}{c}42.1 \% \\
8\end{array}$ & $\begin{array}{c}52.6 \% \\
10\end{array}$ & $\begin{array}{c}47.4 \% \\
9\end{array}$ & $\begin{array}{c}0 \% \\
0\end{array}$ \\
\hline $\begin{array}{l}\text { Más de cuatro sesio- } \\
\text { nes }(n=9)\end{array}$ & $\begin{array}{c}0 \% \\
0\end{array}$ & $\begin{array}{c}33.3 \% \\
3\end{array}$ & $\begin{array}{c}66.7 \% \\
6\end{array}$ & $\begin{array}{c}33.3 \% \\
3\end{array}$ & $\begin{array}{c}44.4 \% \\
4\end{array}$ & $\begin{array}{c}22.2 \% \\
2\end{array}$ \\
\hline $\begin{array}{l}\text { Menos de cuatro años } \\
(n=16)\end{array}$ & $\begin{array}{c}6.3 \% \\
1\end{array}$ & $\begin{array}{c}43.8 \% \\
7\end{array}$ & $\begin{array}{c}50 \% \\
8\end{array}$ & $\begin{array}{c}37.5 \% \\
6\end{array}$ & $\begin{array}{c}50 \% \\
8\end{array}$ & $\begin{array}{c}12.5 \% \\
2\end{array}$ \\
\hline $\begin{array}{l}\text { Cuatro años o más } \\
(n=12)\end{array}$ & $\begin{array}{c}8.3 \% \\
1\end{array}$ & $\begin{array}{l}41.7 \% \\
5\end{array}$ & $\begin{array}{c}50 \% \\
6\end{array}$ & $\begin{array}{l}58.3 \% \\
\quad 7\end{array}$ & $\begin{array}{l}41.7 \% \\
5\end{array}$ & $\begin{array}{c}0 \% \\
0\end{array}$ \\
\hline
\end{tabular}

Tabla 3 Contingencia de resiliencia y dependencia en modalidad individual sin deportes combate y sexo/edad/sesiones de entreno/experiencia deportiva $(\%, \mathrm{~N})$.

\begin{tabular}{lcccccc}
\hline & \multicolumn{3}{c}{$\begin{array}{c}\text { Resiliencia deportes de modalidad } \\
\text { individual sin combate }\end{array}$} & \multicolumn{2}{c}{ EDS-R Dependencia al ejercicio } \\
\hline & <121. Poca & 121-147. Moderada & $>147$. Elevada & $\begin{array}{c}\text { Asintomático no } \\
\text { dependiente }\end{array}$ & $\begin{array}{c}\text { Sintomático no } \\
\text { dependiente }\end{array}$ & $\begin{array}{c}\text { Riesgo de } \\
\text { dependencia }\end{array}$ \\
\hline Hombres $(n=60)$ & $16.7 \%$ & $61.7 \%$ & $21.7 \%$ & $50 \%$ & $43.3 \%$ & $6.7 \%$ \\
& 10 & 37 & 13 & 30 & 26 & 4 \\
Mujeres $(n=24)$ & $16.7 \%$ & $54.2 \%$ & $29.2 \%$ & $45.8 \%$ & $50 \%$ & $4.2 \%$ \\
& 4 & 13 & 7 & 11 & 12 & 1 \\
Junior $(n=31)$ & $12.9 \%$ & $71 \%$ & $16.1 \%$ & $38.7 \%$ & $51.6 \%$ & $9.7 \%$ \\
& 4 & 22 & 5 & 12 & 16 & 3 \\
Senior $(n=53)$ & $18.9 \%$ & $52.8 \%$ & $28.3 \%$ & $54.7 \%$ & $41.5 \%$ & $3.8 \%$ \\
Hasta cuatro & 10 & 28 & 15 & 29 & 22 & 2 \\
sesiones $(n=33)$ & $24.2 \%$ & $63.6 \%$ & $12.1 \%$ & $48.5 \%$ & $48.5 \%$ & $3 \%$ \\
Más de cuatro & 8 & 21 & 4 & 16 & 16 & 1 \\
sesiones $(n=51)$ & $11.8 \% 6$ & $56.9 \%$ & $31.4 \%$ & $49 \%$ & $43.1 \%$ & $7.8 \%$ \\
Menos de cuatro & $8.9 \%$ & $64.3 \%$ & $26.8 \%$ & $46.4 \%$ & $48.2 \%$ & 4 \\
años $(n=56)$ & 5 & 36 & 15 & 26 & 27 & $5.4 \%$ \\
Cuatro años o más & $32.1 \% 9$ & $50 \%$ & $17.9 \%$ & $53.6 \%$ & $39.3 \%$ & $7.1 \%$ \\
$(n=28)$ & 14 & 5 & 15 & 11 & 2 \\
\hline
\end{tabular}

En cuanto a la edad, no se hallaron diferencias en los niveles de resiliencia en los deportistas categorizándolos por junior-senior en ninguno de los distintos deportes, en la línea de un estudio previo en hockey para el que no aparecían diferencias (Tutte \& Reche, 2016) y de fútbol, balonmano, esquí y snowboard (Castro Sánchez, Chacón Cuberos, Zurita Ortega \& Espejo Garcés, 2016). No se han encontrado diferencias en ningún grupo de deportes en función de las sesiones de entrenamiento semanales y se ha observado cómo en deportes individuales se encuentra una significativa menor resiliencia a medida que van pasando los años de experiencia. Se puede revelar entonces que el desarrollo deportivo en el juego de los deportes individuales (sin incluir deportes de combate) presenta cierta menor necesidad 
Tabla 4 Contingencia de resiliencia y dependencia en modalidad colectivo y sexo/edad/sesiones de entreno/experiencia deportiva $(\%, \mathrm{~N})$.

\begin{tabular}{|c|c|c|c|c|c|c|}
\hline & \multicolumn{3}{|c|}{ Resiliencia deportes de modalidad colectiva } & \multicolumn{3}{|c|}{ EDS-R Dependencia al ejercicio } \\
\hline & $<$ 121. Poca & 121-147. Moderada & $>$ 147. Elevada & $\begin{array}{c}\text { Asintomático } \\
\text { no dependiente }\end{array}$ & $\begin{array}{l}\text { Sintomático no } \\
\text { dependiente }\end{array}$ & $\begin{array}{c}\text { Riesgo de } \\
\text { dependencia }\end{array}$ \\
\hline Hombres $(n=113)$ & $\begin{array}{c}26.5 \% \\
30\end{array}$ & $\begin{array}{c}54 \% \\
61\end{array}$ & $\begin{array}{c}19.5 \% \\
22\end{array}$ & $\begin{array}{c}23.9 \% \\
27\end{array}$ & $\begin{array}{c}69.9 \% \\
79\end{array}$ & $\begin{array}{c}6.2 \% \\
7\end{array}$ \\
\hline Mujeres $(n=53)$ & $\begin{array}{c}13.2 \% \\
7\end{array}$ & $\begin{array}{c}50.9 \% \\
27\end{array}$ & $\begin{array}{c}35.8 \% \\
19\end{array}$ & $\begin{array}{c}28.3 \% \\
15\end{array}$ & $\begin{array}{c}64.2 \% \\
34\end{array}$ & $\begin{array}{c}7.5 \% \\
4\end{array}$ \\
\hline Junior $(n=99)$ & $\begin{array}{c}26.3 \% \\
26\end{array}$ & $\begin{array}{c}53.5 \% \\
53\end{array}$ & $\begin{array}{c}20.2 \% \\
20\end{array}$ & $\begin{array}{c}25.3 \% \\
25\end{array}$ & $\begin{array}{c}66.7 \% \\
66\end{array}$ & $\begin{array}{c}8.1 \% \\
8\end{array}$ \\
\hline Senior $(n=67)$ & $\begin{array}{c}16.4 \% \\
11\end{array}$ & $\begin{array}{c}52.2 \% \\
35\end{array}$ & $\begin{array}{c}31.3 \% \\
21\end{array}$ & $\begin{array}{c}25.4 \% \\
17\end{array}$ & $\begin{array}{c}70.1 \% \\
47\end{array}$ & $\begin{array}{c}4.5 \% \\
3\end{array}$ \\
\hline $\begin{array}{l}\text { Hasta cuatro } \\
\text { sesiones }(n=138)\end{array}$ & $\begin{array}{c}22.5 \% \\
31\end{array}$ & $\begin{array}{c}55.1 \% \\
76\end{array}$ & $\begin{array}{c}22.5 \% \\
31\end{array}$ & $\begin{array}{c}26.1 \% \\
36\end{array}$ & $\begin{array}{c}68.1 \% \\
94\end{array}$ & $\begin{array}{c}5.8 \% \\
8\end{array}$ \\
\hline $\begin{array}{l}\text { Más de cuatro } \\
\text { sesiones }(n=28)\end{array}$ & $\begin{array}{c}24.4 \% \\
6\end{array}$ & $\begin{array}{c}42.9 \% \\
12\end{array}$ & $\begin{array}{c}35.7 \% \\
10\end{array}$ & $\begin{array}{c}21.4 \% \\
6\end{array}$ & $\begin{array}{c}67.9 \% \\
19\end{array}$ & $\begin{array}{c}10.7 \% \\
3\end{array}$ \\
\hline $\begin{array}{l}\text { Menos de cuatro } \\
\text { años }(n=149)\end{array}$ & $\begin{array}{l}23.5 \% \\
35\end{array}$ & $\begin{array}{c}51 \% \\
76\end{array}$ & $\begin{array}{c}25.5 \% \\
38\end{array}$ & $\begin{array}{c}24.8 \% \\
37\end{array}$ & $\begin{array}{c}67.8 \% \\
101\end{array}$ & $\begin{array}{c}7.4 \% \\
11\end{array}$ \\
\hline $\begin{array}{l}\text { Cuatro años o más } \\
(n=17)\end{array}$ & $\begin{array}{c}11.8 \% \\
2\end{array}$ & $\begin{array}{c}70.6 \% \\
12\end{array}$ & $\begin{array}{c}17.6 \% \\
3\end{array}$ & $\begin{array}{l}29.4 \% \\
5\end{array}$ & $\begin{array}{c}70.6 \% \\
12\end{array}$ & $\begin{array}{c}0 \% \\
0\end{array}$ \\
\hline
\end{tabular}

de respuestas flexibles cambiantes que los colectivos o deportes de combate, repercutiendo en su resiliencia y favoreciendo respuestas más ajustadas al ambiente. En cualquier caso, sería interesante conocer el clima motivacional de los deportistas, lo cual puede ofrecer un significado, ya que como se sabe, un clima orientado a la tarea desarrolla la resiliencia en los atletas (Chacón-Cuberos et al., 2019).

Por otro lado, la prevalencia del riesgo de dependencia al ejercicio físico se ve representada por el $6.5 \%$ de los participantes en el estudio, datos similares a otros estudios recientes, como el de Reche et al., (2019), con un $8.3 \%$. No se han encontrado diferencias por sexo en ninguna modalidad seleccionada, en la línea de investigaciones con deportistas de modalidad colectiva, individual y de combate (Reche et al., 2015; Reche et al., 2019; Reche-García, Montero \& Martínez-Rodríguez, 2018); en deportistas de modalidad colectiva e individual (Cabrita, Rosado, De la Vega \& Serpa, 2018); y de forma específica, en estudios con corredores y practicantes de ejercicio (Edmunds, Ntoumanis \& Duda, 2006; Furst \& Germone, 1993) y culturistas (Smith \& Hale, 2004), corredores, nadadores, ciclistas y triatletas (Latorre et al., 2016); en corredores, ciclistas, duatletas y triatletas (Ortiz \& Arbinaga, 2017); y en atletas de ironman (Magee, Buchanan \& Barrie, 2016). Estos resultados difieren de lo encontrado por Weik y Hale (2009), quienes hallaron diferencias que señalaban que los hombres mantenían mayor riesgo de sintomatología relacionada con dependencia al ejercicio físico medida con el EDS-R, y diferencias que sugerían que eran las mujeres las que presentaban mayor riesgo siendo evaluadas con el EDQ y el DFT, que miden diferentes dimensiones de la dependencia al ejercicio físico. $Y$ a lo referido por quienes apuntan diferencias en los hombres con una sintomatología mayor, posiblemente debida a las características de la muestra, estudiantes universitarios, que no asegura su participación deportiva (Hausenblas \& Downs, 2002). Así también, a lo referido previamente por Masters y Lambert (1989); Pierce, Rohaly y Fritchley, (1997), y Summers, Machin y Sargent (1983), para los que las mujeres tienen más probabilidades de ser dependientes del ejercicio que los hombres, lo cual genera un campo de debate y reflexión, ya señalado, sobre la precisión en los métodos de investigación y el uso de la escala más adecuada para permitir una mejor comparabilidad de la prevalencia entre las distintas disciplinas deportivas (Di Lodovico, Poulnais \& Gorwood, 2019).

En cuanto a la edad, no se encontraron diferencias en el riesgo de dependencia al ejercicio físico de los deportistas evaluados en función de pertenecer a la categoría junior (de 18 a 20 años) o senior (de 20 en adelante). Parece haber cierta controversia en cuanto a la edad de aparición del trastorno por dependencia al ejercicio físico. Por edad, la mayoría de las investigaciones respaldan que la dependencia al ejercicio físico influye más sobre aquellos deportistas más jóvenes (Allegre, Therme \& Griffiths, 2007; Edmunds et al., 2006; González-Cutre \& Sicilia, 2012; Lindwall \& Palmeira, 2009; Szabo, Frenkl \& Caputo, 1996), y entre los menores de 18 años (Reche-García et al., 2018); sin embargo, otros no encuentran asociaciones (Furst \& Germone, 1993; Klein et al., 2004).

En este estudio no se obtuvieron diferencias en función de la dedicación deportiva ni en función de los años de experiencia deportiva conforme a lo referido antes (Reche-García et al., 2018), y en divergencia con estudios que aseveran que deportistas con una alta dedicación (más de cuatro horas semanales) presentan una media global más elevada de los síntomas consistentes con una 
dependencia al ejercicio físico frente a los que realizan menos de cuatro horas semanales en una muestra de deportistas de ámbito universitario (Reche, Martínez-Rodríguez \& Ortín, 2015). A priori, por la especificidad de la muestra o deportes evaluados, o ambos. Siguiendo la bibliografía más actualiza$\mathrm{da}$, el aumento del volumen de entrenamiento puede ser un factor de riesgo de dependencia al ejercicio físico para tener en cuenta (pudiendo ser favorecido o no por deportes de competición como correr a larga distancia), aunque no se puede discernir si este es una causa o una consecuencia de este trastorno (Valenzuela \& Arriba-Palomero, 2017), que también puede ser favorecido por factores individuales. Lo que sí se afirma es que el hecho de mayor dedicación deportiva provoca un estrechamiento de las potencialidades de realización de la persona, lo que puede causar una dependencia al ejercicio físico, perjudicando el bienestar psicológico y social (Berczik et al., 2012; Chen, 2016).

Como propuestas futuras, sería necesario establecer protocolos para prevención o detección temprana de la dependencia al ejercicio físico en función de lo descrito en este estudio, y difundir las bondades de los deportes de combate frente al resto de las modalidades deportivas.

Se plantea la necesidad de difundir esta información en la comunidad, promoviendo salud, previniendo la aparición de posibles trastornos y fortaleciendo al individuo; y entre psicólogos del deporte, permitiendo una mejor focalización de las estrategias de intervención.

A pesar de la amplia participación del número de deportistas en este trabajo, el tamaño de la muestra utilizado podría ser, en cierto modo, una limitación de estudio, provocado en parte por la búsqueda de homogeneidad en los participantes del estudio. Para próximas investigaciones se propone incluir más participantes de deportes de combate, y no solo de las disciplinas más representativas. Además, entre los deportistas que practican un deporte individual (sin combate) se podrían diferenciar en deportistas con oponente o sin él.

\section{Conclusiones}

De los resultados mostrados, de su análisis y discusión, se pueden obtener las siguientes conclusiones sobre la resiliencia y dependencia al ejercicio físico en función del tipo de deporte (colectivo, individual sin combate, deporte de combate):

1) La sintomatología de riesgo de dependencia al ejercicio físico en los deportes de combate en todas sus dimensiones, menos la que refiere a síntomas de abstinencia, es menor frente a los deportes de equipo e individuales (sin incluir los deportes de combate). Se refiere a un riesgo de malestar clínicamente significativo en diversas áreas de la vida del deportista, con posibles manifestaciones psicopatológicas, físicas y sociales; con consecuencias adversas y repetidas relacionadas con su práctica deportiva y su retirada; y que afectan su calidad de vida, bienestar y rendimiento en su deporte.

2) La resiliencia, o dicho de otro modo, la capacidad de cambiar de forma flexible distintas respuestas para adecuarlas al ambiente, que presentan los participantes de deportes de combate, está por encima de deportistas de modalidades colectivas o individuales (sin incluir deportes de combate). Prevalecen sentimientos de autoconfianza, independencia, decisión, invencibilidad, poderío, ingenio y perseverancia.

3) Los deportistas que practican algún deporte individual. Sin incluir los deportes de combate, presentan menores niveles de resiliencia en función de su desarrollo y años de experiencia deportiva, quizás por la menor necesidad (en comparación con el resto de modalidades analizadas) de respuestas flexibles cambiantes, o de factores intrínsecos de estos deportes.

4) Las deportistas de equipo presentan mayores niveles de resiliencia que los hombres, afrontando la adversidad con respuestas menos rígidas que ellos, siendo más sensibles a las características de su deporte y a la necesidad de integración de recursos individuales combinados.

\section{Referencias}

Allegre, B., Therme, P., \& Griffiths, M. (2007). Individual factors and the context of physical activity in exercise dependence: A prospective study of 'ultra-marathoners'. International Journal of Mental Health and Addiction, 5(3), 233-243. https://doi. org/10.1007/s11469-007-9081-9

American Psychiatric Association (1994). Diagnostic and Statistical Manual of Mental Disorders (DSM-IV). Washington DC: American Psychiatric Association.

Berczik, K., Szabó, A., Griffiths, M. D., Kurimay, T., Kun, B., Urbán, R., \& Demetrovics, Z. (2012). Exercise addiction: symptoms, diagnosis, epidemiology, and etiology. Substance Use \& Misuse, 47(4), 403-417. https://doi.org/10.3109/10826084.2011.639120

Blaydon, M. J., \& Lindner, K. J. (2002). Eating disorders and exercise dependence in triathletes. Eating Disorders, 10(1), 49-60. https://doi.org/10.1080/106402602753573559

Cabrita, T., Rosado, A., De la Vega, R., \& Serpa, S. (2018). Athletic identity and athlete characteristics as predictor of exercise dependence. Revista de Psicología del Deporte, 27(2), 99-106.

Campos, G. G., Valdivia-Moral, P., Zagalaz, J. C., Ortega, F. Z., \& Romero, O. (2017). Influencia del control del estrés en el rendimiento deportivo: la autoconfianza, la ansiedad y la concentración en deportistas. Retos. Nuevas Tendencias en Educación Física, Deporte y Recreación, 32, 3-6.

Castro Sánchez, M., Chacón Cuberos, R., Zurita Ortega, F., \& Espejo Garcés, T. (2016). Niveles de resiliencia en base a modalidad, nivel y lesiones deportivas. Retos: Nuevas Tendencias en Educación Física, Deporte y Recreación, 29, 162-165.

Chacón-Cuberos, R., Castro-Sánchez, M., Espejo-Garcés, T., \& Zurita-Ortega, F. (2016). Estudio de la resiliencia en función de la modalidad deportiva: fútbol, balonmano y esquí. Retos: Nuevas Tendencias en Educación Física, Deporte y Recreación, 29, 157-161.

Chacón-Cuberos, R., Castro-Sánchez, M., Pérez-Turpin, J. A., Olmedo-Moreno, E. M., \& Zurita-Ortega, F. Z. (2019). Levels of physical activity are associated with the motivational climate and resilience in university students of Physical Education from Andalucía: An explanatory model. Frontiers in Psychology, 10, 1821. https://doi.org/10.3389/fpsyg.2019.01821

Chen, W. J. (2016). Frequent exercise: A healthy habit or a behavioral addiction? Chronic Diseases and Translational Medicine, 2(4), 235-240. https://doi.org/10.1016/j.cdtm.2016.11.014

Di Lodovico, L., Poulnais, S., \& Gorwood, P. (2019). Which sports are more at risk of physical exercise addiction: A systematic review. Addictive Behaviors, 93, 257-262. https://doi.org/10.1016/j.addbeh.2018.12.030 
Downs, D. S., Hausenblas, H. A., \& Nigg, C. R. (2004). Factorial validity and psychometric examination of the Exercise Dependence Scale-Revised. Measurement in Physical Education and Exercise Science, 8(4), 183-201. https://doi.org/10.1207/ s15327841mpee0804_1

Edmunds, J., Ntoumanis, N., \& Duda, J. L. (2006). Examining exercise dependence symptomatology from a self-determination perspective. Journal of Health Psychology, 11(6), 887-903. https://doi.org/10.1177/1359105306069091

Friborg, O., Barlaug, D., Martinussen, M., Rosenvinge, J. H., \& Hjemdal, O. (2005). Resilience in relation to personality and intelligence. International Journal Methods Psychiatric Research, 14(1), 29-42.

Furst, D. M., \& Germone, K. (1993). Negative addiction in male and female runners and exercisers. Perceptual and Motor Skills, 77(1), 192-194. https://doi.org/10.2466/pms.1993.77.1.192

González-Hernández, J. (2011). Percepción de bienestar psicológico y competencia emocional en niveles intermedios de la formación deportiva en deportes de combate. E-balonmano.com: Revista de Ciencias del Deporte, 7, 75-80.

González-Cutre, D., \& Sicilia, A. (2012). Exercise dependence in Spanish users of fitness centres: Differences according to gender, age and practiced activities. Behavioral Psychology/Psicología Conductual, 20(2), 349-364.

Hausenblas, H. A., \& Downs, D. S. (2002). Relationship among sex, imagery and exercise dependence symptoms. Psychology ofAddictive Behaviors, 16(2), 169-172. https://doi. org/10.1037/0893-164X.16.2.169

Hernández-García, R., \& Torres-Luque, G. (2011). Preparación física integrada en deportes de combate. E-balonmano.com: Revista de Ciencias del Deporte, 7, 31-38.

Iglesias, X., Gasset, A., González, C., \& Anguera, M. (2010). Interacción competitiva y presión ambiental en deportes de combate: aplicación de la metodología observacional. Revista Iberoamericana de Psicología del Ejercicio y el Deporte, 5(2), 267-282.

Instituto Nacional de Estadística (2019). Anuario de Estadísticas Deportivas 2019. Ministerio de Cultura y Deporte: Secretaría General Técnica. Subdirección General de Atención al ciudadano, Documentación y Publicaciones.

Klein, D. A., Bennett, A. S., Schebendach, J., Foltin, R. W., Devlin, M. J., \& Walsh, B. T. (2004). Exercise "addiction" in anorexia nervosa: model development and pilot data. Cns Spectrums, 9(7), 531-537.

Latorre, P. Á., Jiménez, A., Párraga, J., \& García-Pinillos, F. (2016). Dependency on physical exercise and body dissatisfaction in various endurance sports and their relation with the motivation to do sports. Revista de Psicología del Deporte, 25(1), 113-120.

Lindwall, M., \& Palmeira, A. (2009). Factorial validity and invariance testing of the Exercise Dependence Scale-Revised in swedish and portuguese exercisers. Measurement in Physical Education and Exercise Science, 13(3), 166-179. https://doi. org/10.1080/10913670903050313

Magee, C. A., Buchanan, I., \& Barrie, L. (2016). Profiles of exercise dependence symptoms in Ironman participants. Psychology of Sport and Exercise, 24, 48-55. https://doi.org/10.1016/j.psychsport.2016.01.005

Manzano-García, G., \& Ayala-Calvo, J. C. (2013). Psychometric properties of Connor-Davison Resilience Scale in a Spanish sample of entrepreneurs. Psicothema, 25(2), 245-251. https://doi. org/10.7334/psicothema2012.183

Márquez, S., \& Vega, R. D. L. (2015). La adicción al ejercicio: un trastorno emergente de la conducta. Nutricion Hospitalaria, 31(6), 2384-2391. https://doi.org/10.3305/nh.2015.31.6.8934

Masters, K. S., \& Lambert, M. J. (1989). On gender comparison and construct validity: An examination of the commitment to running scale in a sample of marathon runners. Journal of Sport Behavior, 12(4), 196-202.
Mata-Ordóñez, F., Sánchez-Oliver, A., \& Domínguez, R. (2018). Importance of nutrition in weight loss strategies in combat sports. Journal of Sport and Health Research. 10(1), 1-12.

Menezes-de Lucena Carvalho, V.A., Fernández-Calvo, B., Hernández-Martín, L., Ramos-Campos, F., \& Contador-Castillo, I. (2006). Resilence and the burnout-engagement model in formal caregivers of the elderly. Psicothema, 18(4), 791-796.

Modolo, V. B., Mello, M. T. D., Giménez, P. R. B. D., Tufik, S., \& Antunes, H. K. M. (2009). Dependência de exercício físico: humor, qualidade de vida em atletas amadores e profissionais; Physical exercise dependence: mood, quality of life in amateur and professional athletes. Revista Brasileira de Medicina do Esporte, 15(5), 355-359. https://doi.org/10.1590/S151786922009000600007

Moreno-Murcia, J.A., Belando, N., Huéscar, E., \& Torres, M.D. (2017). Social support, physical exercise and life satisfaction in women. Revista Latinoamericana de Psicología, 49(3), 149-202. https://doi.org/10.1016/j.rlp.2016.08.002

Morgan, P. B., Fletcher, D., \& Sarkar, M. (2013). Defining and characterizing team resilience in elite sport. Psychology of Sport and Exercise, 14(4), 549-559.

Ortiz, C., \& Arbinaga, F. (2017). Dependencia y psicopatología en deportistas: un estudio piloto. Información Psicológica, 112. https://doi.org/10.14635/IPSIC.2016.112.4

Pierce, E. F., Daleng, M. L., \& McGowan, R. W. (1993). Scores on exercise dependence among dancers. Perceptual and Motor Skills, 76(2), 531-535. https://doi.org/10.2466/pms.1993.76.2.531

Pierce, E. F., Rohaly, K. A., \& Fritchley, B. (1997). Sex differences on exercise dependence for men and women in a marathon road race. Perceptual and Motor Skills, 84(3), 991-994. https://doi. org/10.2466/pms.1997.84.3.991

Pierce, E. F., \& Morris, J. T. (1998). Exercise dependence among competitive power lifters. Perceptual and Motor Skills, 86(3), 1097-1098. https://doi.org/10.2466/pms.1998.86.3.1097

Reche, C., De Francisco, C., Martínez-Rodríguez, A., \& Ros-Martínez, A. (2018). Relación entre variables sociodemográficas y deportivas, dependencia del ejercicio y burnout: un estudio preliminar en deportistas. Anales de Psicología, 34(2), 398-404.

Reche, C., Gómez-Díaz, M., Martínez-Rodríguez, A., \& Tutte, V. (2018). El optimismo como contribución a la resiliencia deportiva. Revista Iberoamericana de Psicología del Ejercicio y el Deporte, 13(1), 131-136.

Reche, C., Martínez-Rodríguez, A., \& Ortín, F. J. (2019). Characterization of the resilience and exercise dependence athlete. Ciencia, Cultura y Deporte, 43(15), 17-23.

Reche, C., Martínez-Rodríguez, A., \& Ortín, M. (2015). Exercise dependence and mood states indicators in university athletes. Cuadernos de Psicología del Deporte, 15(2), 21-26. https:// doi.org/10.4321/S1578-84232015000200003

Reche, C., \& Ortín, (2013). Consistencia de la versión española de la escala de resiliencia en esgrima. Avances de la Psicología del Deporte en Iberoamérica, 2(2), 49-57.

Reche, C., Tutte, V., \& Ortín, F. J. (2014). Resiliencia, optimismo y burnout en judokas de competición uruguayos. Revista lberoamericana de Psicología del Ejercicio y el Deporte, 9(2), 19-31.

Reche-García, C., Montero, F. O., \& Martínez-Rodríguez, A. (2018). Aspects related with physical exercise dependence in university students. Sport TK-Revista EuroAmericana de Ciencias del Deporte, 7(2), 39-44.

Richardson, J. T. (2011). Eta squared and partial eta squared as measures of effect size in educational research. Educational Research Review, 6(2), 135-147.

Ruiz-Barquín, R. (2012). Relaciones entre características de personalidad y estados de ánimo: un estudio con deportistas de combate universitarios. Revista Iberoamericana de Psicología del Ejercicio y el Deporte, 7(1), 89-112. 
Ruiz-Barquín, R., De la Vega-Marcos, R., Poveda, J., Rosado, A., \& Serpa, S. (2012). Psychometric analysis of Resilence Scale in the sport football. Revista de Psicología del Deporte, 21(1), 143-151.

Salgado, A. C. (2005). Métodos para medir la resiliencia: Una alternativa peruana. Liberabit. Revista de Psicología, 11, 41-48.

Sicilia, Á., Alías-García, A., Ferriz, R., \& Moreno-Murcia J. A. (2013). Spanish adaptation and validation of the Exercise Addiction Inventory (EAl). Psicothema, 25(3), 377-383. https:// doi.org/10.7334/psicothema2013.21

Sicilia, A., \& González-Cutre, D. (2011). Dependence and physical exercise: Spanish validation of Exercise Dependence Scale Revised (EDS-R). The Spanish Journal of Psychology, 14(1), 421- 432.

Smith, D. K., \& Hale, B. D. (2004). Validity and factor structure of the bodybuilding dependence scale. British Journal of Sports Medicine, 38(2), 177-181. https://doi.org/10.1136/bjsm.2002.003269

Summers, J. J., Machin, V. J., \& Sargent, G. I. (1983). Psychosocial factors related to marathon running. Journal of Sport Psychology, 5(3), 314-331. https://doi.org/10.1123/jsp.5.3.314

Szabo, A., Frenkl, R., \& Caputo, A., (1996). Deprivation feelings, anxiety, and commitment in various forms of physical activity: A cross-sectional study on the internet, Psychologia, 39, 223-230.

Tutte, V., \& Reche, C. (2016). Burnout, resiliencia y optimismo en el hockey sobre hierba femenino. Cuadernos de Psicología del Deporte, 16(3), 73-78.
Valenzuela, P. L., \& Arriba-Palomero, F. (2017). Risk of exercise addiction among male amateur triathletes and its relationship with training variables. Revista Internacional de Ciencias del Deporte, 13(48), 162-171. https://doi.org/10.5232/ricyde2017.04806

Wagnild, G. M., \& Young, H. M. (1993). Development and psychometric evaluation of resilience scale. Journal of Nursing Measurement, 1(2), 165-178.

Waugh, C. E., Thompson, R. J., \& Gotlib, I. H. (2011). Flexible emotional responsiveness in trait resilience. Emotion, 11, 1059-1067. https://doi.org/10.1037/a0021786

Weik, M., \& Hale, B. D. (2009). Contrasting gender differences on two measures of exercise dependence. British Journal of Sports Medicine, 43(3), 204-207. https://doi.org/10.1136/ bjsm.2007.045138

Zurita-Ortega, F., Chacón-Cuberos, R., Cofre-Bolados, C., Knox, E., \& Muros, J. J. (2018). Relationship of resilience, anxiety and injuries in footballers: Structural equations analysis. PloS One, 13(11), e0207860. https://doi.org/10.1371/journal.pone.0207860 